\title{
PARTO VAGINAL INSTRUMENTADO EN EL HOSPITAL UINIVERSITARIO SAN VICENTE DE PAÚL DURANTE UN PERÍODO DE 5 AÑOS (2000-2004), MEDELLÍN, COLOMBIA
}

\section{Operative vaginal delivery over a 5-year period (2000-2004) at the San Vicente de Paúl teaching hospitall in Medellín, Colombia}

Hernán Cortés, M.D.* Eugenio Escobar, M.D. **

Recibido: octubre 10/2005 - Revisado: enero 24/2006 - Aceptado: febrero 21/2006

\section{RESUMEN}

Objetivo: determinar el porcentaje de partos instrumentados en el Hospital Universitario San Vicente de Paúl, durante un periodo de 5 años y caracterizar los partos en relación al tipo de fórceps utilizados, indicaciones y técnica empleada.

Materiales y Métodos: estudio descriptivo retrospectivo, realizado en el Hospital Universitario San Vicente de Paúl del 2000 - 2004, donde se incluyeron todos los partos atendidos en este periodo.

Resultados: se atendieron 9.255 partos, de los cuales el 2\% (185), fueron instrumentados con fórceps. El fórceps más usado fue el de Kielland y el 63\% fueron fórceps bajos y de desprendimiento. Se presentaron desgarros grados III y IV en el 19\% de las pacientes sometidas a parto instrumentado.

Conclusiones: se presentó una incidencia baja de parto instrumentado y está disminuyendo, la incidencia de desgarros fue baja en relación con otras

* Docente Departamento de Obstetricia y Ginecología Universidad de Antioquia, ginecoobstetra Universidad de Antioquia. Ginecólogo Cooperativa SOGOS, Hospital Universitario San Vicente de Paúl. Correo electrónico: hcortes@mixmail.com

** Ginecólogo Cooperativa SOGOS, Hospital Universitario San Vicente de Paúl, ginecoobstetra Universidad de Antioquia. series. Se debe estimular la enseñanza y práctica del arte de los fórceps.

Palabras clave: fórceps obstétrico, parto vaginal instrumentado, desgarro perineal, Colombia

\section{SUMMARY}

Objective: determining the percentage of operative vaginal deliveries at the San Vicente de Paúl teaching hospital over a five-year period and characterising operative deliveries in relation to the type of forceps, indications, and techniques used.

Methods: descriptive retrospective study carried out at the hospital 2000-2004, including all deliveries during this period.

Results: 9,255 deliveries were included; 2\% (185) of them were operative vaginal deliveries. Kielland forceps were those most used, 63\% being outlet and low forceps. Nineteen percent of patients suffered grade III and IV perineal tears.

Conclusions: a low incidence of operative vaginal deliveries was observed; this is decreasing. The incidence of tears was low in relation to other series. The teaching and practice of the art of using forceps should be encouraged. 


\section{INTRODUCCIÓN}

En todo el mundo la tasa de cesáreas continua en aumento, en EE.UU. la tasa es de aproximadamente un $25 \%$, pero en países como Brasil y Costa Rica puede llegar a ser tan alta como un 50\%; este aumento se debe no solo al aumento de intervenciones primarias (en nulíparas), sino también por el número de cesáreas iterativas. Este aumento se presenta de manera concomitante con la disminución en el uso de fórceps. ${ }^{1}$

En EE.UU. la frecuencia de partos instrumentados se estima en un 10\%, la mayoría de estos por medio de extracción obstétrica por aspiración (vacuum), mientras el fórceps se utiliza más o menos en un 3\% de los casos, igualmente en el Reino Unido, las tasas de parto instrumentado varían entre un 10-15\%, con preferencia en el uso de la extracción obstétrica por aspiración (vacuum). ${ }^{2}$

El uso de fórceps ha disminuido principalmente por el aumento en el número de cesáreas cuando se espera un parto difícil, por razones medicolegales y por un menor entrenamiento en las escuelas de obstetricia.

Sin embargo existen situaciones en las cuales el fórceps tiene una indicación superior a la cesárea o el uso de extracción obstétrica por aspiración (vacuum), a saber: ${ }^{3,4}$

- Extracción de la cabeza en el parto en podálica

- Parto asistido del bebe pretérmino (< 34 semanas)

- Presentación de cara

- Sospecha de coagulopatía o trombocitopenia en el bebé

- Contraindicación materna para el pujo

- Anestesia general

- Bloqueo epidural

- Mal pujo o fatiga materna

- Sufrimiento fetal agudo en el expulsivo, entre otras.

Motivo por el cual el obstetra debe estar entrenado en su uso e indicaciones, teniendo en cuenta que la cesárea tiene mayores riesgos para la madre en algunas situaciones (cardiopatías, coagulopatías, etc.) y para el bebé (retención de cabeza en podálica, sufrimiento fetal agudo); además, en ocasiones cuando no se pudiera realizar una cesárea en tan corto tiempo, por el personal e instalaciones que se requieren (quirófano ocupado, anestesiólogo en otro servicio, etc.), es de anotar también que el parto con fórceps aumenta las posibilidades de otro parto vaginal en comparación con la cesárea.,

Sin embargo los fórceps se han asociado con un aumento en la morbilidad materna, principalmente al trauma del piso pélvico (desgarros 3 y 4, incontinencia urinaria y rectal), mayor sangrado y dolor; igualmente se asocia a mayor morbilidad neonatal (hemorragia intracraneal, fracturas, céfalohematomas, etc.), la cual disminuye a medida que se incrementa la experiencia del operador. ${ }^{7,8}$

Los objetivos del presente trabajo son determinar el porcentaje de partos instrumentados en el Hospital Universitario San Vicente de Paúl, durante el periodo 2000 - 2004, describir el tipo de fórceps usados, las indicaciones, la técnica empleada y la incidencia de desgarros perineales III y IV.

\section{MATERIALES Y MÉTODOS}

Aspectos éticos: Se tuvieron en cuenta las consideraciones de la resolución 8430 del 83 y se contó con la aprobación del comité de ética de la institución, Se garantizó la confidencialidad de los datos.

Cohorte retrospectiva en la que la población de referencia se basó en todos los partos atendidos en el Hospital Universitario San Vicente de Paúl en el periodo 2000 - 2004. Este es un hospital general de alta complejidad, centro de referencia de la región noroccidental de Colombia. Además, se estudiaron los desenlaces en todas las pacientes expuestas a parto instrumentado en dicho periodo. Los datos fueron tomados de los libros de procedimientos del servicio de urgencias obstétricas de la institución (partos espontáneos, cesáreas y fórceps) y de las historias clínicas en las pacientes en las que se usaron los fórceps. Variables a medir: presencia de parto vaginal espontáneo, cesáreas, partos instrumentados, tipo de fórceps usado, indicación, técnica empleada, complicaciones maternas (desgarros III y IV), tipo 
de anestesia, apgar del recién nacido.

Análisis de la información: Los datos obtenidos se presentaron por medio de porcentajes.

\section{RESULTADOS}

Durante este periodo se atendieron en el Hospital Universitario San Vicente de Paúl (HUSVP) 9.255 partos, de los cuales 185 (2\%) se intervinieron con fórceps, se realizaron 3.715 cesáreas $(40,1 \%)$ y se atendieron 5.355 partos (57,9\%).

Solo se presentó un caso de fórceps fallido que terminó por cesárea. No fue posible revisar cinco historias clínicas. La tabla 1 muestra la distribución de los partos por año. El tipo de fórceps más utilizado fueron los de Kielland (tabla 2)

Las indicaciones para el uso del fórceps fueron: expulsivo prolongado (41 casos), sufrimiento fetal agudo (15 casos), mal pujo (21 casos), cardiopatía (12 casos) y otras causas en 10 casos. En relación con la técnica empleada el 50\% de los casos fueron fórceps bajos, el 13\% de desprendimiento, el 8\% fórceps medios y solo un caso de fórceps en podálica, en el 28\% de los casos no se describió la técnica. En cuanto a la anestesia, la técnica conductiva (raquídea y epidural) fue utilizada en el $82 \%$ de los casos, en el 13\% de los casos se requirió anestesia general y en el 5\% solo se utilizó anestesia local.

Se presentaron desgarros en el 19\% de las pacientes (34 casos), 14\% fueron desgarros grado III y $5 \%$ grado IV. Es de anotar que en 55 pacientes (30\%) no se realizó episiotomía.

Con respecto al resultado neonatal, 26 recién nacidos $(14,4 \%)$ tuvieron un puntaje de apgar al mi-

\section{Tabla 2. Tipos de fórceps utilizados}

Kielland $165(91,6 \%)$

Simpson 3

Espátulas de Velasco

2

Piper

Sin datos 1 9

nuto menor o igual a 4, a los cinco minutos 13 recién nacidos $(7,2 \%)$ presentaron un puntaje de apgar menor o igual a 6. Hubo dos mortinatos, uno con malformaciones severas y el otro por abrupción placentaria; de 2 recién nacidos no se obtuvieron datos.

\section{DISCUSIÓN}

En el HUSVP la incidencia de parto instrumentado es baja al compararla con la frecuencia del uso de fórceps en otros países y en algunas regiones de EE.UU., por ejemplo en Escocia y EE.UU. la incidencia es cercana al 10\%, mientras en Noruega es del 8\%; en EE.UU. la incidencia del uso de fórceps varía en los diferentes estados, entre un 20\% a 2,1\%, sin tener en cuenta el uso de la extracción obstétrica por aspiración (vacuum). ${ }^{1} \mathrm{Al}$ mismo tiempo, el uso de fórceps en el HUSVP está disminuyendo, pues se pasó de 2,9\% en el 2000 al 1,2\% en el 2004.

Llama la atención que el fórceps más usado sea el de Kielland (91,6\%), en comparación con el de Simpson (1,6\%), ya que este último es el mas usado en el mundo. ${ }^{10}$

Con respecto a la técnica, el $88 \%$ fueron fórceps bajos o de desprendimiento, que en general se consideran "fáciles" y no requieren de una técnica ni un

\begin{tabular}{|c|c|c|c|c|c|}
\hline & 2000 & 2001 & 2002 & 2003 & 2004 \\
\hline Partos & $1.377(56 \%)$ & $1.256(56,2 \%)$ & 1. $135(60,9 \%)$ & $579(55,6 \%)$ & $1.008(60,3 \%)$ \\
\hline Cesáreas & $1.002(41 \%)$ & $929(41,6 \%)$ & $698(37,4 \%)$ & $444(42,7 \%)$ & $642(38,4 \%)$ \\
\hline Fórceps & $70(2,9 \%)$ & $46(2 \%)$ & $31(1,66 \%)$ & $17(1,63 \%)$ & $21(1,2 \%)$ \\
\hline
\end{tabular}


entrenamiento tan depurado; llama de igual manera la atención que en el 28\% de los casos no se describa la técnica empleada, conociendo las implicaciones medicolegales que puede conllevar su uso.

La incidencia de desgarros III y IV, los cuales causan la mayor morbilidad en la madre fue del 19\%, esta se puede considerar como baja en comparación con lo reportado por Hankins y Kabiru quienes presentan una incidencia en sus series del $27 \mathrm{y}$ el $39 \%$ respectivamente. ${ }^{1,8}$

Para concluir y como lo afirma Friedman "el arte del fórceps tiende a desaparecer", ${ }^{11}$ no obstante, mientras existan partos y se apliquen analgesias epidurales, se requerirá el uso de fórceps y la única manera de disminuir los riesgos que este conlleva es mantener un buen nivel de entrenamiento y experiencia, por lo tanto, se debe estimular la enseñanza y la práctica de este arte, principalmente en los centros de formación.

\section{AGRADECIMIENTOS}

Al personal de archivo del Hospital San Vicente de Paúl, por la valiosa colaboración en la búsqueda de las historias clínicas.

\section{REFERENCIAS}

1. Hankins GD, Rowe TF. Operative vaginal deliveryyear 2000. Am J Obstet Gynecol 1996;175:275-82.

2. Bofill JA, Rust OA, Perry KG, Roberts WE, Martin RW, Morrison JC. Operative vaginal delivery: a survey of fellows of ACOG. Obstet Gynecol 1996;88:1007-10.

3. Patel RR, Murphy DJ. Forceps delivery in modern obstetric practice. BMJ 2004;328:1302-5.

4. Parto vaginal instrumental. En: Cunningham F, MacDonald P, Gant N, et al. Williams Obstetricia. Ed. $20^{\circ}$. Panamericana, 1997. p. 443-462.

5. Bahl R, Strachan B, Murphy DJ. Outcome of subsequent pregnancy three years after previous operative delivery in the second stage of labour: cohort study. BMJ 2004;328:311.

6. Murphy DJ, Liebling RE. Cohort study of maternal views on future mode of delivery following operative delivery in the second stage of labor. Am J Obstet Gynecol 2003;188:542-8.

7. Murphy DJ, Liebling RE, Verity L, Swingler R, Patel R. Early maternal and neonatal morbidity associated with operative delivery in the second stage of labour: a cohort study. Lancet 2001;358:1203-7.

8. Kabiru WN, Jamieson D, Graves W, Lindsay M. Trends in operative vaginal delivery rates and associated maternal complication rates in an inner city hospital. Am J Obstet Gynecol 2001;184:1112-4.

9. Notzon FC, Bersgjo P, Cole S, Irgens LM, Daltveit AK. International collaborative effort (ICE) on birth weight, perinatal and infant mortality. Differences in obstetrical delivery practice. Norway, Scotland and the United States. Acta Obstet Gynecol Scand 1991;70:451-60.

10. Ross MG, Beall MH, Bonni A. Forceps delivery; 2004. [Visitado en 2005] Disponible en: www.emedicine. com $/ \mathrm{med} /$ topic 3284

11. Parto con Fórceps. En: Gilstrap LC, Cunningham FG, Van Dorsten JP. Urgencias en Sala de Partos y Obstetricia Quirúrgica. 2a ed. Ed. Panamericana; 2004. p. 89121.

Conflicto de intereses: Este artículo se realizó con el apoyo económico de la cooperativa SOGOS,

de la cual hacen parte los dos autores. Esta es una cooperativa de trabajo asociado que presta servicio asistencial en el área de urgencias de ginecología y obstetricia del Hospital San Vicente de Paúl. 